\title{
WOLFGANG SCHENKEL
}

\section{Die Farben in ägyptischer Kunst und Sprache}

\section{A Einleitung}

Noch immer unbefriedigend beantwortet ist die Frage nach der Verwendung der einzelnen Farben in der ägyptischen Malerei. Grundsätzlich ist nur klar, daß das Verfahren der Bemalung in älterer Zeit, auf jeden Fall im AR, im wesentlichen die Polychromie ist, nicht die Koloristik (vgl. Wolf, Die Kunst Ägyptens, 267f.). Im folgenden wird nun versucht, die Farbverwendung in der Malerei unter Berücksichtigung der naturwissenschaftlichen Faktoren aus den Sprachmitteln zu erhellen, mit denen die ägyptische Sprache die Farbwelt faßt. Ein solches Vorgehen erscheint gerechtfertigt, wenn man die zentralen Begriffe der Kunstbetrachtung Schäfers über Wolf hinaus konsequent weiterführt: von der Wesenhaftigkeit der Kunst und der Darstellung dessen, was der Künstler in seiner Vorstellung trägt (in Schäfers Sinn, vgl. Von ägyptischer Kunst, 1930³, 86; 94; 97 f.), kommt man über den Gegenstandsbezug der Kunst und die Darstellung von Denkbildern (Wolf, Die Kunst Ägyptens, 272; 278f.; 660f. und öfter) ohne Bruch zur Sprache als Ort der geistigen Gegenstände und der Denkbilder, wie sie die inhaltbezogene Sprachwissenschaft herausgearbeitet hat. Im besonderen soll hier an die wirkungbezogene Sprachbetrachtung L. Weisgerbers ${ }^{1}$ angeknüpft wẹrden. Die Frage, die sich stellt, ist dann, inwieweit sich die Besonderheiten der Farbverwendung in der Malerei als Wirkungen der ägyptischen Sprache verstehen lassen. Sollte sich ein solches Vorgehen als brauchbar erweisen, so öffnete sich hier grundsätzlich ein Zugang zum Verständnis der Eigengesetzlichkeit der ägyptischen Kunst aus der ihr zugeordneten ägyptischen Sprachgemeinschaft heraus, da sich die Verbreitung von ägyptischer Kunst und Sprache räumlich und zeitlich im wesentlichen decken.

Um möglichst deutliche Ergebnisse zu gewinnen, wird die Untersuchung in der Hauptsache auf das AR eingeschränkt; erstens liegt hier genügend Material bei der Hand, das leicht verarbeitet werden kann: Ransom Williams, The Decoration of the Tomb of Per-nēb, 1932, 38 - 74, und Smith, A History of Egyptian Sculpture and Painting in the Old Kingdom, 19492, 257-263, besonders die Zeichenliste, 366-382; zweitens ist zu erwarten, daß die gesuchten Beziehungen zwischen Sprache und Kunst in älterer Zeit enger und damit leichter erkennbar sind als in späterer.

\section{B Die Farben in der Malerei}

Im AR malt der ägyptische „Künstler“ im wesentlichen sechsfarbig. Mit unseren Farbwörtern sind diese sechs Farben am besten so zu benennen: „Weiß" (vgl. Lucas, Ancient Egyptian Materials and Industries, 19483, 399); "Rot" (Lucas, 397-399); „Gelb“ (Lucas, 399f.); ,Grün“ (Lucas, 396f.; Per-nēb, 25f.); „Blau“ (Lucas, 392-395; Per-nēb, 26-31); und „Schwarz“ (Luc as, 391 f.). Die sonst noch bekannten Farben sind selten, z. T. im AR nicht vorhanden. So ist „Rosa" (Lucas, 397) erst im NR belegt. „Braun“" und „Grau“ (Lucas, 395 f.; 397) kommen zwar im AR vor (s. auch Per-nēb, 25), sind jedoch sehr viel seltener als die sechs „Grundfarben“. Auch die von Lucas, 414, untersuchte Palette aus dem Grab des Tutenchamun hat nur diese sechs Farben (,Blau“ ist zwar nicht erhalten, aber fast mit Sicherheit zu ergänzen); eine Palette aus der Zeit Amenophis' II. (Hayes, BMMA 7, Oktober 1948, 60) hat zwar 8 Farbnäpfe, aber nur 6 verschiedene Farben: „Rot", ,Schwarz", „Weiß", „Rot" (nur Spuren), „Blau“, „Grün“, „Gelb“, „Rot". Zeitweilig scheinen sogar noch weniger als sechs Farben für die normalen Bedürfnisse ausgereicht zu haben: so spielte vor der Erfindung des ,,ägyptischen Blau" und dessen geläufiger Verwendung (etwa von der 5. Dynastie an) das ,Blau“" in der Malerei keine Rolle (Per-nēb, 29-31;

1) S. vor allem L. Weisgerber, Die Muttersprache im Aufbau unserer Kultur, 19572; zum Thema Kunst besonders S. 208-212; dabei auch ein erwägenswertes, aber noch genauer zu untersuchendes Beispiel aus der ägyptischen Kunst (S. 210). 
vgl. 54f.); dann zeigen die von Dunham genau beschriebenen Stelen aus Nagc ed-Dêr, daß hier "Blau" und "Grün" lediglich Varianten sind, im ganzen also nur fünf „Grundfarben" vorliegen (vgl. unten Abschn. R).

Einzelne Farben können aus verschiedenen Substanzen hergestellt werden, so daß also chemisch mehr als sechs "Grundfarben" vorliegen. Dies spielt jedoch hier keine Rolle, da die chemisch verschiedenen Stoffe praktisch die gleiche ,Grundfarbe“" liefern: es kommt für uns nur darauf an, daß die "Grundfarben" sich innerhalb ein und derselben Darstellung deutlich von einander abheben. Auch brauchen Farbmischungen und Übermalungen nicht berücksichtigt zu werden: sie sind im AR nicht üblich (vgl. Per-nēb, 36f.) und erst recht bei den Hieroglyphen, die unten als Hauptmaterial verarbeitet werden, nicht zu erwarten.

Die Sechszahl der hier in der Hauptsache behandelten Farben ist auch nicht durch moderne Einteilungsprinzipien bestimmt, da sich diese Farben tatsächlich innerhalb desselben Zusammenhanges klar voneinander abheben; dagegen führt kein Versuch, die Farben weiter in Nuancen zu unterteilen, zu brauchbaren Ergebnissen (vgl. Per-nēb, 36f.). Daß im folgenden nur die sechs "Grundfarben" behandelt werden, wird das Ergebnis nicht beeinflussen: die "Nebenfarben" sind nur wegen der ziemlich geringen Zahl von Belegen weggelassen worden (Zu den „Nebenfarben“" vgl. auch unten Abschn. Q).

\section{Die Farbwechsel in der Malerei}

Wären diese sechs ,Grundfarben“ nun jeweils auf bestimmte Objekte der Darstellung festgelegt, wäre also ein Gegenstand A immer mit der Farbe I, ein Gegenstand B immer mit der Farbe II belegt, so gäbe es keine Schwierigkeiten in der Deutung: die Malerei wäre dann eben realistisch, so weit es die Farbskala und das Prinzip der Polychromie erlauben. Die Aufgabe wäre lediglich noch, die gemalten Gegenstände mit ihren Vorbildern in der Natur zu verbinden.

Leider liegen die Verhältnisse nicht so einfach: die Farben für ein und denselben Gegenstand wechseln, und zwar in einem Maß, daß dies auf Zufall und Nachlässigkeit allein nicht beruhen kann. Tatsächlich wird also ein Gegenstand A bald mit der Farbe I, bald mit der Farbe II belegt; ein Gegenstand B hier mit der Farbe II, dort mit der Farbe I. Bei Ransom Williams und Smith findet man etwa folgende Angaben:

Wechsel

$$
\begin{aligned}
& \text { "Rot" - „Gelb" } \\
& \text { "Gelb" - ,Grün“ } \\
& \text { „Grün" - „Blau“ } \\
& \text { „Blau" - ,Schwarz" } \\
& \text { „Grün" - ,Schwarz" }
\end{aligned}
$$$$
\text { „,common" (Smith, 258); }
$$$$
\text { "seems to have been confined to fibrous objects" (Smith, 258; }
$$$$
\text { im einzelnen s. Per-nēb, 66-69); }
$$$$
\text { Per-nēb, 62ff.; nach Smith, 258, ist dieser Wechsel häufiger }
$$$$
\text { als Ransom Williams annahm; }
$$$$
\text { ,the most common interchange in the Old Kingdom" (Smith, }
$$$$
\text { 258; zum einzelnen s. Per-nēb, 54ff.); }
$$$$
\text { Smith, 258; Per-nēb, 62, Anm. } 152 .
$$

Dazu kommen noch extremere Wechsel, so wenn Kupfer zwar „,blau“ gemalt wird, Kupferspiegel aber gewöhnlich ,gelb“ oder „rot" (Per-nēb, 53). Hier liegt jedoch sicher der Unterschied bereits in den Vorbildern. Damit ist die Liste der Farbwechsel zwar noch nicht erschöpft, aber die wichtigsten sind notiert.

\section{Bisherige Erklärung der Farbwechsel in der Malerei}

Wie ist dieser merkwürdige, überaus häufige Farbwechsel zu erklären? Ransom Williams, die sich ausführlich mit diesem Problem befaßt, sucht so weit wie möglich die verschiedeneFarbbehandlung auf verschiedenfarbige Vorlagen in der Natur zurückzuführen. Dies gelingt, wenn auch nicht immer ganz überzeugend, bei relativ selten belegten Farbwechseln: im Einzelfall findet sich eben meist noch eine Erklärungsmöglichkeit. Dies geht aber nicht mehr, wenn die Zahl der Belege steigt, und so ist sie gezwungen, sich beim Wechsel „Blau" - „Schwarz" nach anderen Ursachen umzusehen. Einmal (Per-nēb 54f.) klingt dabei die Möglichkeit an, daß das „Blau“ 
als zuletzt entdeckte „Grundfarbe“ der Ägypter sich noch nicht ganz durchgesetzt hatte. Doch schließlich kommt sie zu einer psychologischen Erklärung: „as we understand the interchanges, they represent in origin, in most cases, different color concepts for the same unvarying object of vision, according as it impressed different artists" (op. cit. 72). An anderen Stellen geht sie sogar so weit, zu vermuten, daß der Grund in physiologischen Bedingungen liege, durch die sich die Ägypter von uns unterschieden hätten. Smith nimmt dies op. cit. 258 auf: „The blue and black interchange is the most common in the Old Kingdom and was probably due to a lack of sharp visual perception between these two coulours." Mit anderen Worten: die Ägypter wären in diesem Bereich farbenblind gewesen.

Die Unzulänglichkeit der bisherigen Erklärungen liegt auf der Hand. Smith hat bereits festgestellt, daß die Farbwechsel außerhalb des bei Ransom Williams schließlich allein übrigbleibenden „Grün"-,,Blau"-,,Schwarz"-Bereichs erheblich häufiger und damit nicht so leicht wegzuinterpretieren sind. Zum andern ist es ganz unwahrscheinlich, daß die Farbempfindung des Ägypters und damit der Bau seines Auges anders gewesen sein soll als bei heutigen Menschen: so schnell verändert sich der Mensch nicht. Dazu kommt, daß man auch in anderen Fällen, wo man zunächst mit Farbenblindheit operierte (z. B. bei der Blaublindheit der Römer), schließlich doch zu anderen Lösungen gefunden hat.

\section{E Statistik zu den Farbwechseln in der Malerei}

Bevor nun irgendwelche Schlußfolgerungen gezogen werden, soll hier eine exaktere Materialbasis gelegt werden, um die Farbwechsel genauer, d. h. quantitativ zu fassen. Dazu bietet sich Smiths Hieroglyphenliste an, da die Zahl der Belege ziemlich hoch ist, hoch genug, um sie statistisch auswerten zu können.

Die folgende Tabelle umfaßt alle bei Smith notierten Farben, auch „Braun“. Nur die mehrfarbigen Hieroglyphen sind nicht aufgenommen. Die wenigen „Grau"-Belege bleiben wie bei Smith unter „Schwarz" stehen. Heller gemalte Zeichen, die von einer dunkleren Farbe des gleichen Farbtons umrandet sind, werden ohne besondere Kennzeichnung unter die Belege des Farbtons summiert. Diese Ungenauigkeiten zu eliminieren, erscheint kaum sinnvoll, wenn man bedenkt, wie exakt die Smithsche Liste, die die Farben ja nur mit unseren alltäglichen und sehr groben Farbadjektiven charakterisiert, ïberhaupt sein kann. Besondere Sorgfalt ist bei „Weiß“ nötig, da es immer eine Umrandung mit einem anderen Farbton hat, entweder eine ,rote" oder eine ,schwarze", was nicht ohne Einfluß auf den Gebrauch sein wird: es erscheint deshalb in drei Rubriken: 1. „Weiß" von „Rot" umrandet (Weiß (R)); 2. „Weiß" von „Schwarz" umrandet (Weiß $(\mathrm{S})$ ) ; 3. ,Weiß" überhaupt (Weiß $(\mathrm{R}+\mathrm{S})$ ). In der ersten waagrechten und senkrechten Zeile stehen die Farben und die Gesamtzahl der mit ihnen gemalten Zeichen. Die übrigen Zahlen geben an, wieviel Zeichen jeweils sowohl mit der links stehenden als auch mit der oben stehenden Farbe belegt sind.

\begin{tabular}{|c|c|c|c|c|c|c|c|c|}
\hline Rot & Gelb & Grün & Blau & Schwarz & Braun & $\begin{array}{l}\text { Weiß } \\
\text { (R) } \\
\text { (18) }\end{array}$ & $\begin{array}{l}\text { Weiß } \\
\text { (S) } \\
(23)\end{array}$ & $\begin{array}{r}\text { Weiß } \\
(\mathrm{R}+\mathrm{S}) \\
(39)\end{array}$ \\
\hline 10 & 13 & 15 & 12 & 9 & 2 & - & - & pou \\
\hline 4 & 7 & 13 & 10 & 8 & 2 & 2 & - & \\
\hline 7 & 7 & 2 & 2 & 2 & 1 & - & & \\
\hline 1 & 3 & 1 & 1 & 1 & - & & & \\
\hline 12 & 10 & 13 & 25 & 一 & & & & \\
\hline 9 & 12 & 23 & - & & & & & \\
\hline 7 & 21 & - & & & & & & \\
\hline 29 & - & & & & & & & \\
\hline
\end{tabular}

Gelb (91)

Rot (81)

Bereits hier ist es rätlich, „Braun“ nicht mehr weiter zu behandeln, da die geringe Zahl von Belegen für eine Statistik unbrauchbar ist. Alle übrigen Farben sind häufiger belegt. Um die 
Materialbasis nicht unnötig zu verkleinern, scheint es geraten, bei allen Farben mit sämtlichen Belegen zu arbeiten, und nicht die Belege für jede Farbe auf die gleiche Zahl durch eine zufällige Auswahl zu reduzieren: man hätte sonst höchstens 39 Belege pro Farbe, falls man ,Weiß" mit „rotem" Rand und „Weiß" mit ,schwarzem" Rand noch auseinander halten wollte, nur höchstens 18. Belege pro Farbe. Um trotzdem zu einigermaßen richtigen Vergleichszahlen zu kommen, die von der Zahl der Belege für die Einzelfarben ziemlich unabhängig sind, wird berechnet, wieviel Prozent der Zeichen eines Farbtons mit einem anderen Farbton wechseln. Dadurch ergeben sich für die Häufigkeit des Wechsels zweier Farben A und B untereinander zwei Prozentzahlen, die eine von $\mathrm{A}$ aus, die andere von B aus berechnet; die eine gibt an, wieviel Prozent der A-Zeichen auch noch mit der Farbe B gemalt werden können, die andere, wieviel Prozent der B-Zeichen auch mit der Farbe A gemalt werden können. In der folgenden Tabelle geht die untere Zahl von den links stehenden Farben aus, die obere von den oben stehenden.

\begin{tabular}{|c|c|c|c|c|c|c|c|}
\hline & Rot & Gelb & Grün & Blau & Schwarz & $\begin{array}{c}\text { Weiß } \\
\text { (R) }\end{array}$ & $\begin{array}{l}\text { Weiß } \\
\text { (S) }\end{array}$ \\
\hline$\therefore$ & 12,35 & 14,29 & 30,61 & 24,49 & 21,43 & - & - \\
\hline Weiß $(R+S)$ & 25,64 & 33,34 & 38,46 & 30,77 & 23,08 & - & - \\
\hline Weiß (S) & $\begin{array}{r}4,94 \\
17,39\end{array}$ & $\begin{array}{r}7,69 \\
30,43\end{array}$ & $\begin{array}{l}26,53 \\
56,52\end{array}$ & $\begin{array}{l}51,02 \\
59,52\end{array}$ & $\begin{array}{l}19,05 \\
34,78\end{array}$ & $\begin{array}{r}11,11 \\
8,70\end{array}$ & - \\
\hline Weiß (R) & $\begin{array}{r}8,64 \\
38,89\end{array}$ & $\begin{array}{r}7,69 \\
38,89\end{array}$ & $\begin{array}{r}4,08 \\
11,11\end{array}$ & $\begin{array}{r}4,08 \\
11,11\end{array}$ & $\begin{array}{r}4,76 \\
11,11\end{array}$ & - & \\
\hline Schwarz & $\begin{array}{l}14,81 \\
28,57\end{array}$ & $\begin{array}{l}10,99 \\
23,81\end{array}$ & $\begin{array}{l}26,53 \\
30,95\end{array}$ & $\begin{array}{l}51,02 \\
59,52\end{array}$ & - & & \\
\hline Blau & $\begin{array}{l}11,11 \\
18,37\end{array}$ & $\begin{array}{l}13,19 \\
24,49\end{array}$ & $\begin{array}{l}46,94 \\
46,94\end{array}$ & - & & & \\
\hline Grün & $\begin{array}{r}8,64 \\
14,29\end{array}$ & $\begin{array}{l}23,08 \\
42,86\end{array}$ & - & & & & \\
\hline Gelb & $\begin{array}{l}35,80 \\
31,87\end{array}$ & & & & & & \\
\hline
\end{tabular}

Rot

Um nun ein Maß für die Vertauschbarkeit zweier Farbtöne zu erhalten, das man auf verschiedene Weise berechnen könnte, wird hier der Durchschnitt der beiden Prozentzahlen gegeben: $\left(\frac{X \cdot 100}{a}+\frac{Y \cdot 100}{b}\right): 2$, wobei $X$ bzw. Y der Prozentsatz der a bzw. b Zeichen der Farben A bzw. B sind, die mit der jeweils anderen Farbe wechseln können. Diese Tabelle sieht dann so aus:

Rot Gelb Grün Blau Schwarz Weiß Weiß Weiß

$\begin{array}{lllllllll}\text { Weiß (R+S) } & 19 & 24 & 35 & 28 & 22 & - & (\mathrm{R}) & (\mathrm{R}+\mathrm{S}) \\ \text { Weiß (S) } & 11 & 19 & 42 & 32 & 27 & 10 & - & - \\ \text { Weiß (R) } & 24 & 23 & 8 & 8 & 8 & - & \\ \text { Schwarz } & 22 & 17 & 29 & 55 & - & \\ \text { Blau } & 15 & 19 & 47 & - & & \\ \text { Grün } & 11 & 33 & - & & & \\ \text { Gelb } & 23 & - & & & & \\ \text { Rot } & - & & & & & \end{array}$


Bereits aus der ersten Liste ergab sich, daß jede Farbe mit jeder wechseln kann. Dabei muß berücksichtigt werden, daß hier alle einfarbigen Zeichen verwendet wurden, ohne Rücksicht darauf, ob durch das Vorbild in der Natur eine Farbe vorgegeben war, oder ob der Ägypter seine Farbe frei erfinden könte. Da diese Fehlerquelle jedoch in allen Fällen wohl etwa gleich groß sein wird, braucht sie hier nicht berücksichtigt zu werden. Eine Ausschaltung dieser Fehler scheint ohnehin äußerst schwierig, da bei vielen Zeichen überhaupt und dann jeweils im Einzelfall, bei der von einem bestimmten Künstler in einem bestimmten Augenblick gemalten Hieroglyphe, schwer entschieden werden kann, ob ein Naturvorbild vorliegt oder nicht. Für unsere Zwecke kommt es aber auch gar nicht darauf an, wie die Farben zu ihren Vorbildern in der Natur stehen, sondern nur darauf, wie sich die einzelnen Farben in der Malerei für den Ägypter zu einander verhalten. Dafür wird die Aufstellung ausreichen.

In die Augen springend ist in der letzten Tabelle, daß die Vertauschbarkeit ganz unterschiedlich groß ist, am größten zwischen „Blau"-,,Schwarz"; „Blau“-,Grün“. Dieser Tatbestand war für Ransom-Williams der eigentliche Ansatzpunkt. Es ist aber voreilig, bereits hier Schlüsse zu ziehen. Nur die hier zuständige Farbenlehre kann die Basis für eine Deutung sichern.

\section{F Farbenlehre}

Das Prinzip des Aufbaus der Farbwelt kann man sich leicht am Modell Ostwalds, dem Doppelkegel, klar machen (Wilhelm Ostwald, Die Farbenlehre, 4 Bde., Leipzig. 1921 ff.; das Modell auch einfach dargestellt und leicht zu greifen in Ostwald, Die Farbenfibel, 5. Abschnitt; hier Fig. 1).

Die (senkrechte) Achse enthält die ,,un-

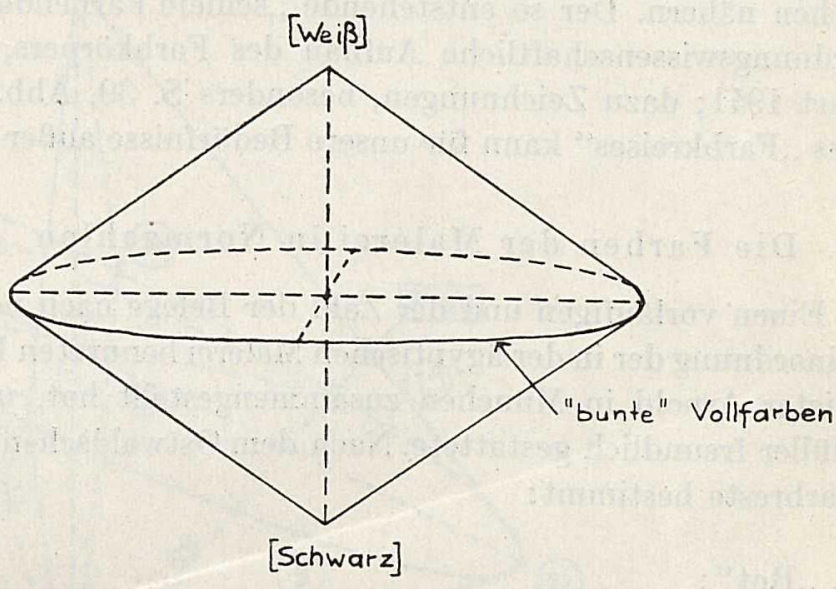

Fig. 1 bunten" Farben von „Weiß" über "Grau" nach ,Schwarz". Auf dem größten Umfang, wo also die beiden Kegel sich berühren, sitzen die „bunten“ „Vollfarben“, (in Ostwalds willkürlicher Benennung) von „,Gelb" zu „,Kreß" (=,Orange“), ,Rot", ,Veil“" (= ,Violett"), ,U(ltramarin)blau“, „Eisblau“, „Seegrün“, „Laubgrün“ und wieder zurück zu „Gelb“. Alle übrigen Farben lassen sich als Mischungen von Vollfarben mit „Weiß" und „Schwarz" darstellen und verteilen sich gesetzmäßig zwischen dem Kreis und der Achse.

Mit diesem Modell wäre sehr einfach zu arbeiten. Leider ist aber die Farbwelt komplizierter aufgebaut: nach dem Modell müßten alle „Vollfarben" die gleiche Helligkeit haben. Daß dies nicht der Fall ist, zeigt bereits ein Blick auf Ostwalds Farbenkreis in der Farbenfibel ${ }^{9}$, 19: bei „Gelb" ist die spezifische Helligkeit am größten, bei „Ublau“ am kleinsten. Die „kalten“ Farben sind dunkler als die „warmen“ (s. Ostwald, Die Farbenlehre I 103; II 199f.; IV 88-93; in Bd. IV ist das Spektrum des Totalfarbenblinden auf ' $T$ af. I anschaulich). „Es handelt sich . . . bei der Helle offenbar um eine mit dem Farbton verkoppelte Eigenschaft" (M. Richter, Grundriß der Farbenlehre der Gegenwart, Dresden u. Leipzig 1940. Wissenschaftliche Forschungsberichte. Naturwissenschaftliche Reihe, Bd. 51, S. 30). Wie stark die Abweichung von Ostwalds Modell ist, zeigt der reizmetrische Luther-Nybergsche Farbkörper (S. Richter, loc. cit. 110-115): die hellste "Vollfarbe" liegt so hoch wie das weiße Ende der Achse, die dunkelste ,Vollfarbe" liegt so tief wie das schwarze Ende der Achse. Der genannte reizmetrische Farbkörper kann für uns natürlich nicht als Modell dienen, da im folgenden nur ein empfindungsgemäßer brauchbar ist.

Ostwalds Anordnung der ,Vollfarben“ auf dem Farbenkreis entspricht nicht ganz der empfindungsgemäßen Ordnung, da er gleichzeitig auf dem Kreis empfindungsgleiche Abstände und dazu kompensative Farbtöne einander gegenüber haben wollte. Beides ist aber nicht zu vereinen: tatsächlich kommt es nur auf die Gleichabständigkeit an (Richter, op. cit. 153-156). Bei „Eis- 
blau“ und „Seegrün“ sind die Abstände im Vergleich zu anderen Bereichen zu klein, wie ein Blick auf den Farbenkreis in der Farbenfibel ${ }^{9}, 19$ anschaulich zeigt.

Trotzdem wird für die weitere Untersuchung der Ostwaldsche Farbenkreis benutzt: ein vollkommen empfindungsgemäßer Farbenkreis ist nur dann notwendig, wenn die zu verarbeitenden Daten die entsprechende Präzision haben. Die ägyptischen Farben, die oben als ,Weiß“, „Rot“ usw. bezeichnet wurden, sind aber auf diese Art nur grob bestimmt und sicher von den „Vollfarben" auf dem Kreis selbst verschieden. Es kommt noch ein weiterer Punkt hinzu: unten wird versucht, an Hand des Ostwaldschen Farbnormensystems einige Angaben zu den Farbtönen einzubeziehen. Diese Daten können aber nur dann unmittelbar in Beziehung zu einander gebracht werden, wenn man sie in das Ostwaldsche Modell einsetzt.

Da wir hier an den Ostwaldschen Doppelkegel gebunden sind, auch kein anderes Modell ebenso leicht benutzt werden kann, muß ein praktischer Notbehelf einspringen: der Farbenkreis wird gegen die Achse gedreht, so daß sich die Helligkeitsverhältnisse der „Vollfarben“ den tatsächlichen nähern. Der so entstehende ,schiefe Farbendoppelkegel“ findet sich bei H. Podestà, Der ordnungswissenschaftliche Aufbau des Farbkörpers, Bücherei des Augenarztes, Heft 9, Stuttgart 1941; dazu Zeichnungen, besonders S. 39, Abb. 8. Die Frage der Kreis- oder Ellipsenform des ,Farbkreises" kann für unsere Bedürfnisse außer acht bleiben.

\section{G Die Farben der Malerei in Normżahlen}

Einen vorläufigen und der Zahl der Belege nach noch ausbaubedürftigen Anhaltspunkt für die Einordnung der in der ägyptischen Malerei benutzten Farben können einige Angaben bieten, die mir Dieter Arnold in München zusammengestellt hat, und deren Benutzung Prof. Hans Wolfgang Müller freundlich gestattete. Nach dem Ostwaldschen Farbnormenatlas, 1923/24 ${ }^{3}$ wurden folgende Farbreste bestimmt:

1. ,Rot":

Hautfarbe der Männer:

5. Dynastie (Staatssammlung 1600): 6 le, an dünneren Stellen 5 lc;

11. Dynastie (Staatssammlung 1618): 6 le;

Amarnazeit (Glyptothek 84): $6 \mathrm{pg}$;

Ramessidisch (Wittelsbacher Ausgleichsfond 25): 5 lc;

In der Hieroglyphe $\odot$ :

11. Dynastie (Staatssammlung 3390): 5 lc.

2. ,Gelb":

11. Dynastie: 3 lc (Staatssammlung 1774); 3 ic (Staatssammlung 1619).

3. „Grün“:

Blätter eines Strauches:

5. Dynastie (Staatssammlung 2921): 21 ie bis 21 ia;

Pflanzenbündel (Schilf ?):

5. Dynastie (Glyptothek 263): 22 ic bis 21 na.

4. „Blau":

Falkenfedern:

11. Dynastie (Staatssammlung 3390): 18 ie; $15 \mathrm{gc} ; 14$ ga;

Wasser:

5. Dynastie (Glyptothek 254): 14 ic; 16 ie.

Zur Erhärtung einiger dieser Werte, zu Korrekturen bei anderen können Farbnormzahlen dienen, die mir Ernst Kühn für die Mineralien, aus denen die ägyptischen Malfarben hergestellt wurden, an 
Stücken der Vorlesungssammlung des Chemischen Instituts und der Sammlung des Mineralogischen Instituts in Heidelberg bestimmt hat. Als Farbtafel wurde die kleine Farbmeßtafel nach Wilhelm Ostwald, Ausgabe A, bearbeitet von Gerhard Streller und Grete Ostwald, GöttingenBerlin 1930, benutzt. E. Kühn schreibt zum Bestimmen der Normzahlen: „Da die Mineralien etwas eingestaubt waren, habe ich jeweils an zwei bis drei Stellen mit dem Messer einen Kristallit abgeschabt und das Pulver verworfen. Dann habe ich erneut etwas Mineral abgeschabt und das nun entstehende Pulver mit der Farbmeßtafel verglichen. Pulver von den verschiedenen Stellen des Mineralbrockens sahen dabei immer gleich aus. Die Genauigkeit der Farbtonnummern glaube ich auf $\pm 1 / 2$ Farbtonnummer genau abgelesen zu haben. Die Genauigkeit der Ablesung der Grauverhüllung schätze ich auf \pm 1 Weiß- und \pm 1 Schwarz-Stufe oder besser. Die Messungen wurden bei Tageslicht (bedeckter Himmel) im Zimmer in der Nähe des Fensters gemacht."

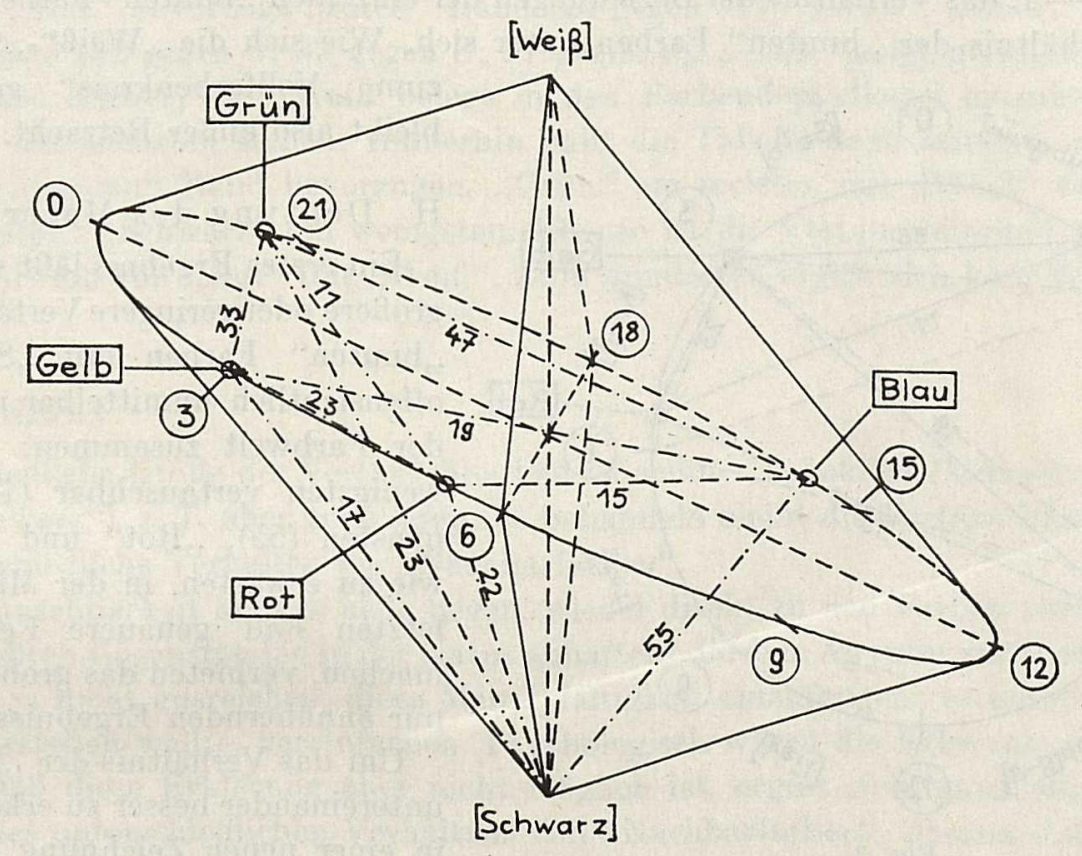

Fig. 2

Ergebnisse (nach „Grundfarben" geordnet; in Klammern stehen jeweils die Fundorte der benutzten Stuicke):

1. „Rot":

Roter Ocker: 6 pe (Irlbach).

2. ,Gelb":

Auripigment (Arsensulfid): 3 na (1) Ungarn; 2) Fundort unbekannt);

Gelber Ocker: 3 pe bis 3 nc (Grube Sulzbach).

3. ,Grün“"

Malachit: 21 ga bis 21 ia (1) Belgisch Kongo; 2) Ural; 3) Fundort unbekannt);

Chrysocoll: 20 lc (Chile).

4. "Blau“:

Azurit: 16 ia bis 16 la (Fundort unbekannt).

(Das Kalzium-Kupfer-Silikat ließ sich nicht auftreiben).

Welche Farbnormen für die ägyptische Malerei mehr zutreffen, die nach den Denkmälern oder die nach den Mineralien bestimmten, ist nicht entscheidbar. In Fig. 2 sind nur die nach den Denkmälern aufgenommen, in Fig. 3 beide. Für die unten vorgebrachte Hypothese zu den ,Nachbarfarben" sind die der Mineralien günstiger, die der Denkmäler aber auch schon hinreichend. 
Die Buchstaben der Normzahlen zu verwerten, ist hier zu schwierig; dazu bewegen sich die Schwankungen in ziemlich engen Grenzen, so daß man also ohne große Verzerrungen der Darstellbarkeit halber die entsprechenden „Vollfarben“ einsetzen kann. Die Zahlen dagegen lassen sich in den veränderten Farbendoppelkegel eintragen (Fig. 2): zur Orientierung sind die Ostwaldschen Farbbezeichnungen in eckigen Klammern z. T. (die übrigen s. in Fig. 3) angegeben. Die ägyptischen Farbstoffe sind nach einem Durchschnittswert der oben nach den Denkmälern gegebenen Farbnormzahlen eingeordnet (umrandet): "Gelb": 3; „Rot": 51/2; „Blau“: 15 1/2, "Grün": $21 \frac{1}{2}$. Da „Weiß“ noch einer Sonderbehandlung bedarf, wird hier das veränderte Modell nur mit den Zahlen für die Wechsel zwischen den übrigen Farben gegeben. Deshalb ist es auch hier unnötig, die Verschiebung nach „Weiß", die bei fast allen Farbbelegen der genannten Denkmäler vorliegt, zu berücksichtigen. Beurteilt wird anschließend - in zwei verschiedenen Arbeitsgängen - 1. das Verhältnis der Beziehungen der einzelnen „bunten“Farben zu „Schwarz" und 2. das Verhältnis der „bunten“ Farben unter sich. Wie sich die „Weiß"-,,Schwarz"-Achse

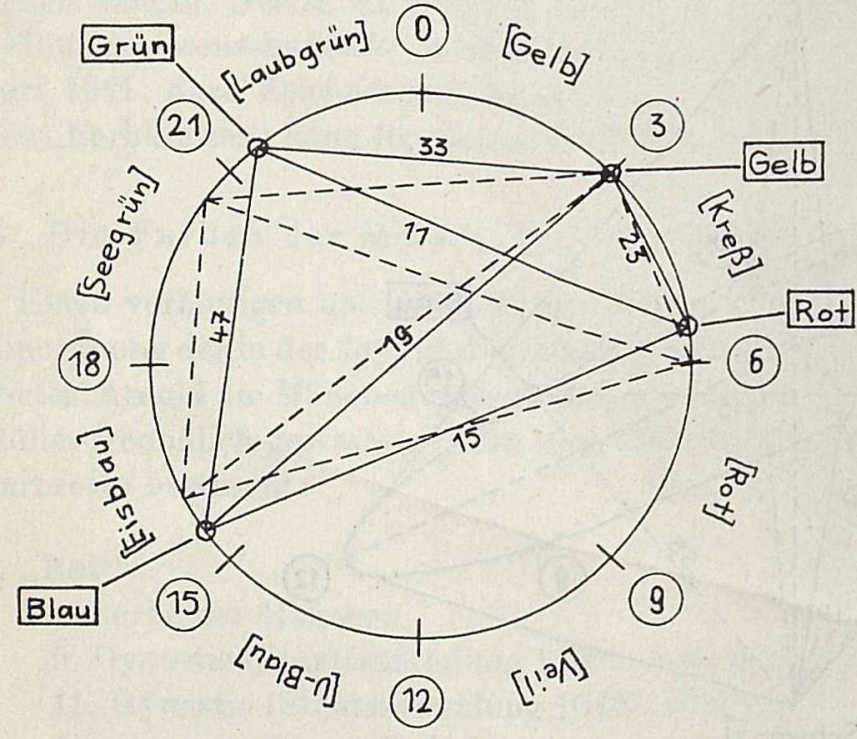

Fig. 3 zum „Vollfarbenkreis" genauer verhält, bleibt also außer Betracht.

\section{H Deutung des Materials}

Ein erstes Ergebnis läßt sich ablesen: die größere oder geringere Vertauschbarkeit der "bunten“ Farben mit „Schwarz" hängt offensichtlich unmittelbar mit dem Aufbau der Farbwelt zusammen: „Gelb“ ist am wenigsten vertauschbar (17), „Blau" am meisten (55), "Rot" und "Grün" liegen, wie zu erwarten, in der Mitte $(22 ; 29)$; im letzten Fall genauere Feststellungen zu machen, verbieten das grobe Modell und die nur annähernden Ergebnisse der Statistik.

Um das Verhältnis der „,bunten“ Farben untereinander besser zu erkennen, wird hier in einer neuen Zeichnung der Farbenkreis allein gegeben (Fig. 3): Angaben wie in Fig. 2; zusätzlich sind die Beziehungen angegeben, die sich bei Zugrundelegung der Normzahlen nach den Mineralien ergeben (gestrichelt): ,Gelb": 3 ; „,Rot": 6; ,Blau“: 16; „Grün“: 201/2.

Theoretisch sollte man nun folgendes erwarten: liegen Nachbar-,,Grundfarben" in ihrem Farbton eng beieinander, so ist die Vertauschbarkeit gering, da nur in wenigen Fällen streitig sein kann, ob die eine oder die andere „Grundfarbe“ für einen bestimmten Gegenstand zu wählen ist; liegen Nachbar-, ,Grundfarben“" in ihrem Farbton dagegen weit auseinander, so vergrößert sich der von beiden gemeinsam zu bestreitende Farbbereich, die Vertauschbarkeit steigt.

Damit stimmt die relativ kleine Vertauschbarkeit zwischen „Gelb" und „Rot" (23) im Vergleich mit der großen Vertauschbarkeit zwischen „Grün“ und „Blau“ (47) überein. Nicht stimmt dazu die geringe Vertauschbarkeit von „Rot" und „Blau" (15) und die von ,Gelb“ und „Grün" (33) im Vergleich mit den beiden gerade genannten Beziehungen. Die Vertauschbarkeit von, grob gesagt, Gegenfarben ist, wie nicht anders zu erwarten, gering: ,, Rot “ — ,Grün“(11); ,Gelb“— ,Blau“(19).

Das unterschiedliche Verhalten von „Rot" - "Gelb"/,Grün" — ,Blau“ und "Gelb" „Grün" $/$, Rot - „Blau“ findet in dem Farbkörper keine Begründung. Die Frage wird unten (Abschn. O) weiter zu behandeln sein. Ausdrücklich sei jedoch bemerkt, daß die für das Verhalten der „bunten“ Farben untereinander aufgezeichnete Beobachtung nicht die einzig mögliche ist. Sie liegt jedoch bereits bei den Farbnormzahlen der Denkmäler nahe und drängt sich bei den Farbnormzahlen der Mineralien noch mehr auf; darüber hinaus ist sie die einzige, für die sich unten eine Erklärung geben läßt. 
Hier ist nun der Vollständigkeit halber das Verhalten von „Weiß“ nachzutragen. Wie oben schon festgestellt, ist „Weiß" leider nur mit einer ,,roten“ oder ,,schwarzen“ Umrahmung belegt. Die Tabelle für die Vertauschbarkeit sieht so aus:

$\begin{array}{lccc} & \text { Weiß }(\mathrm{R}) & \text { Weiß }(\mathrm{R}+\mathrm{S}) & \text { Weiß }(\mathrm{S}) \\ \text { Rot } & 24 & 19 & 11 \\ \text { Gelb } & 23 & 24 & 19 \\ \text { Grün } & 8 & 35 & 42 \\ \text { Blau } & 8 & 28 & 32 \\ \text { Schwarz } & 8 & 22 & 27\end{array}$

Aus der Tabelle erkennt man leicht, daß die Vertauschbarkeit des „Weiß“ von der Rahmungsfarbe abhängt: ,Rot" bevorzugt ,,roten" Rand (24 gegen 11); ,Grün“, „Blau“ und „Schwarz" „schwarzen“ Rand (42 gegen 8; 32 gegen 8; 27 gegen 8). „Gelb" neigt geringfügig zu „rotem“" Rand. Es ist also schwer, die "Weiß"belege in den Farbendoppelkegel einzubeziehen. "Gelb" müßte „Weiß" am nächsten stehen. Immerhin paßt die Tabelle dazu insofern, als bei den drei Farben, die ,schwarzen“ Rand bevorzugen, „Grïn“ am meisten mit „Weiß" wechselt, „Blau“ am zweithäufigsten; ,Schwarz" am wenigsten. Ebenso ist die Vertauschbarkeit von „Gelb" mit „Weiß" höher als die von „Rot" mit „Weiß“. Zum mindesten ergibt sich kein Widerspruch zum Modell.

\section{Zwischenbilanz}

Die unterschiedliche Größe der Vertauschbarkeit ist somit z.T. geklärt (Verhältnis der „,bunten“ Farben zu „Schwarz"), z. T. aber noch offen: zu behandeln bleibt die Vertauschbarkeit überhaupt und das unterschiedliche Verhalten bei „Nachbarfarben“.

Bei der Vertauschbarkeit mag es nahe liegen, wieder direkt zu den Farben zurückzugehen, wie sie den dargestellten Gegenständen in der Natur anhaften: die dem Ägypter zur Verfügung stehende Palette konnte ja nicht ausreichen, diese Mannigfaltigkeit einzufangen; er mußte in jedem Fall, wo er etwas darstellen wollte, vereinfachen. Psychologisch wären die Schwankungen dann leicht verständlich. Daß diese Erklärung aber nicht möglich ist, ergibt sich, ganz abgesehen von der Schwierigkeit des unterschiedlichen Verhaltens von „Nachbarfarben“, daraus, daß die ägyptische Kunst stärker polychrom ausgerichtet ist, als die technischen Möglichkeiten fordern: „Nebenfarben" sind zwar herstellbar, werden aber kaum verwendet. So ist es klar, daß der Maler sich nicht in erster Linie mit den Vorbildern in der Natur auseinandersetzt, auch in der Regel nicht die Farben der Naturvorbilder einmal so und einmal anders behandelt, sondern daß er mit einer Farbwelt fertig werden muß, die man nur in der Sprache ansetzen kann. Die nächste Aufgabe ist damit gestellt: die Behandlung der Farben in der Sprache zu untersuchen.

\section{J Allgemeines zu den Farbwörtern}

Die sprachlichen Verfahren des Umgangs mit der Farbwelt herauszuarbeiten, heißt in erster Linie, die Farbwörter aufsuchen. Zuvor ist es aber nützlich, einige Bemerkungen über die Farbwörter überhaupt vorauszuschicken. Der naive Sprachbenutzer - das ist im Normalfall jeder neigt dazu, die Farbwörter der Muttersprache als richtig, d. h. als der Natur der Sache angemessen zu empfinden. Genaueres Zusehen zeigt aber, daß es grundsätzlich verschiedene Verfahren des Umgangs mit der Farbwelt gibt: so gibt es, um nur die beiden wichtigsten Unterschiede zu nennen, Sprachen, die nur sachgebundene Farbwörter haben (z. B. das homerische Griechisch), und andere, die daneben und vor allem abstrakte Farbwörter besitzen, die auf jedes beliebige Objekt angewandt werden können, und die sich zu einem lückenlosen Erfassen der Farberscheinungen zusammenschließen.

Zum andern stehen die Farbwörter zwar in einem Zusammenhang mit den physikalischen und physiologischen Gegebenheiten, können aus ihnen aber nicht abgeleitet werden. Welchen Inhalt 
z. B. das deutsche „Blau“ hat, ergibt sich einzig und allein aus seiner Stellung innerhalb der deutschen abstrakten Farbwörter, aus dem deutschen Farben-,,Feld“. Eine ausführliche Besprechung der Farbwörter findet man bei L. Weisgerber, Vom Weltbild der deutschen Sprache, 1. Halbband 2 , 127-130, und besonders im 2. Halbband ${ }^{2}$, 83-88. Der folgende Abschnitt hat die Aufgabe, die ägyptischen Farbwörter für das AR zusammenzustellen, und weiter zu fragen, ob die ägyptische Sprache abstrakte Farbwörter kennt.

K Die ägyptischen Farbwörter

Die Farbwörter des Ägyptischen werden hier nach den Angaben des Wb. zusammengestellt. Für unsere Frage ist es nur von Bedeutung, den Wortbestand aufzunehmen, den man für das AR erwarten darf. Ziel ist, abstrakte Farbwörter von sachbezogenen zu trennen.

1. , Rot"

dšr ,,rot, rot sein“, Wb. V 288, 1-290, 13; dazu mehrere nominale Ableitungen, Wb. V $290 \mathrm{ff}$. und das Kausativum sdšr Wb. IV 372, 1-3.

tms „rot (besonderer Nuance)“ Wb. V 369, 7-12; dazu verschiedene nominale Ableitungen $369,14$.

$\underline{t r}$,,das Rote, als Bezeichnung für das Blut"Wb. V 386, 13; dazu ein Mineral $t r$ Wb. V 386, 11 bis 12. Wb. V 386, 14-15 können hierher gehören, bleiben aber unsicher.

ins ,das rote Blut" Wb. I 100, 4; dazu mehrere Ableitungen, s. Alliot, RdE 10 (1955) 1-7; ein jnsj genanntes Textil Wb. I 100, 6-13.

$j d m j$ und jdmj.t ,Leinenstoff von roter Farbe“ Wb. I 153, 14-18.

Sonst sind keine Farbwörter mehr unter „Rot" belegt. Zwei Farbstoffbezeichnungen von anderen Wortstämmen ( $j p$ Nä Wb. I, 68, 13-14; jmзß NR Wb. I 80, 16-17) können hier unberücksichtigt bleiben, da sie zu spät belegt sind. Zu mrš Wb. II 113, 1, das erst in griechischer Zeit belegt ist, für unseren Zweck also nicht mehr in Frage kommt, vgl. unten Abschn. I.

Befinden sich in der Liste abstrakte Farbwörter?

Ohne weiteres kann man $j d m j$ ausschneiden (und damit auch $j d m j . t$ ): erstens bezieht sich dieser Wortstamm nur auf ein Textil, wäre also sachgebunden, zweitens ist der jdmj-Stoff gar nicht auf die rote Farbe festgelegt; s. Kees, Farbensymbolik, 463f.; 477.

Alle übrigen Wörter können sich auf das Blut beziehen ( $d \check{s} r ; \underline{t} m s ; \underline{t r}$ und jns). So ist jns nur einmal außerhalb der jnsj-Textilbezeichnung belegt: in PT 1464a, offenbar vom Blut. Aber aus der gleichen Stelle geht hervor, daß jns sehr nahe bei $d \check{s} r . w$,Blut" steht, wie $d \check{s} r$ überhaupt am häufigsten verwendet wird, wenn vom Blut die Rede ist: s. besonders die Formen $d \check{r} r$. $w$ Wb V 491, $9-13$ und $d \check{s} r . t \mathrm{~Wb} . \mathrm{V} 492,1-2$. Offenbar liegt bei jns als Blut eine vereinzelte Übertragung des an den jnsj-Stoff gebundenen Farbwortes vor. Erst in späterer Zeit wird diese Verbindung mit dem Blut weiter ausgebaut, aber auch nicht auf andere Farbträger ausgeweitet. Siehe im einzelnen Alliot, RdE 10 (1955) $1-7$.

Ähnlich liegen die Verhältnisse bei $\underline{t} r$ (eher wohl $\underline{t} w r$, das dann in griechischer Zeit als wtr belegt ist: Wb. I 381, 3; s. Alliot, RdE 10,4). Auch tr ist nur in der Bezeichnung einer Sache zu finden, diesmal eines Minerals (Wb. V 386, 11-12), und dann für das Blut: PT 451c; 1263c; weiter Wb. V 386, 13; $\underline{t r}$. wt , die Blutröte" Kees, Farbensymbolik, 451. Es liegt also sicher kein abstraktes Farbwort vor, die Übertragung auf das Blut bleibt vereinzelt.

Etwas weiter ist der Anwendungsbereich von $\underline{t} m s$. In PT 911a wird es von der sonst $d \grave{r} r . t$ genannten Krone gebraucht. Hier und in PT 1349a kommt es neben dšr, wohl als Variante vor. Bemerkenswert sind nun die Stellen des medizinischen P. Smith, wo tsm erklärt werden muß, also zur Zeit der Anfügung der Glossen nicht mehr ohne weiteres verständlich war:

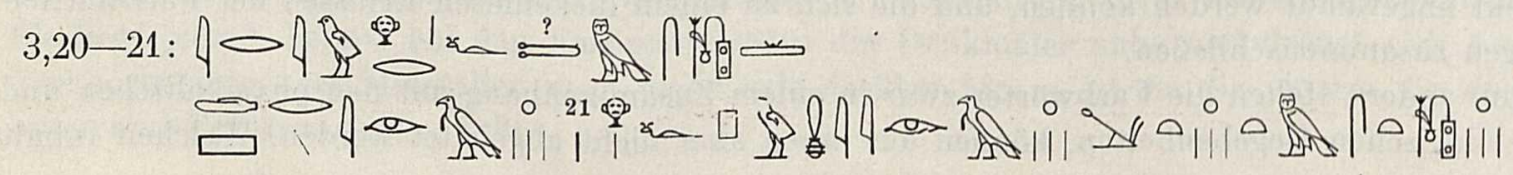


"Was anbetrifft: sein Gesicht ist, ,gerötet".

Das bedeutet: ,rot" ist die Färbung seines Gesichtes wie die Färbung der Frucht der tsm.t-Pflanze."

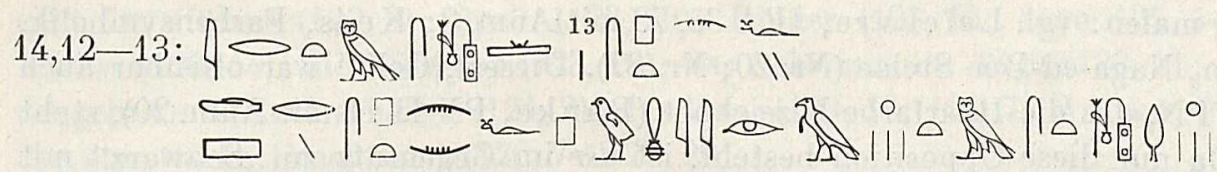

„Was anbetrifft: ,gerötet" sind ihre (der Wunde) beiden Lippen. Das bedeutet: „rot" sind ihre beiden Lippen wie die Färbung des $\underline{t}$ sm.t-Baumes."

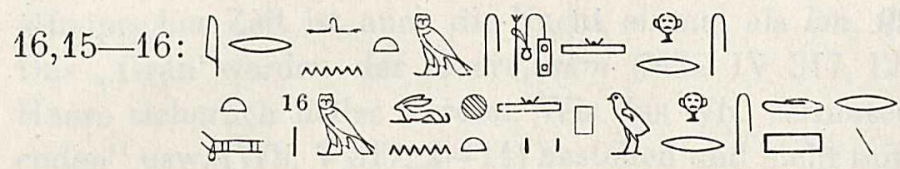

„Was anbetrifft: nicht gibt es eine „Rötung" darauf. Das bedeutet: daß nicht etwas „Rotes" darauf ist."

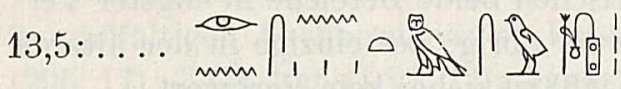

,,sie (die Geschwüre) haben „Rötungen“ gemacht."

Dies wird in der Glosse in 13, 11 aufgenommen mit:

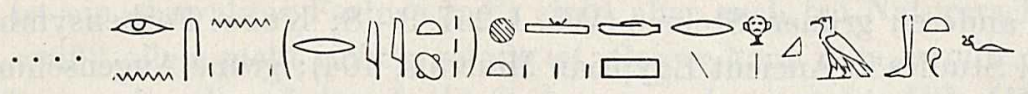

"Sie haben (Eiter) gemacht 〈und > etwas ,Rotes" auf seiner Brust."

Möglicherweise läßt sich nach den medizinischen Symptomen die Farbe tsm bestimmen. Breasted, The Edwin Smith Surgigal Papyrus, 194, zitiert Dr. Luckhardt: „I am inclined to the view that the color meant is the one medical men have in mind when they say that the person is ,cyanotic'. It is a mixture of red and a blue with the blue more in evidence, particularly as the „cyanosis" increasis in severity. The purplish discoloration in this condition is due essentially to a deficient oxygenation of the blood."

Auf die sšd-Binde bezieht sich $\underline{t} s m$ in PT 1147a. tsm.t ist ein roter Stoff: Wb. V 369, 13. Sonst kommt nur noch $\underline{t s m . w}$ in übertragener Bedeutung als „Böses, Unheil“, u. a. vor. (Wb. V 370, $2-6)$, wie das auch bei $d \check{r} r$ häufig ist.

Gleichgültig, ob die medizinische Bestimmung der Farbe stimmt oder nicht, für unsere Frage

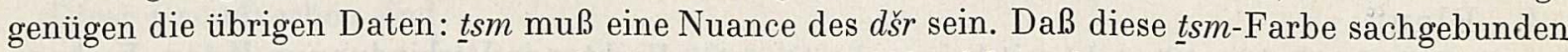
war und nur selten übertragen wurde, ist deutlich; es läßt sich auch vermuten, mit welcher Sache sie zusammenhängt: „Le déterminatif se réfère manifestement à la pastille d'ocre rouge de la palette des scribes, dont l'épithète $d \breve{r} r$ désignerait aussi bien, sinon mieux, la nuance exacte." (Lef ebvre, JEA 35 (1949) 74f.) Da aber auch $\underline{t} r$ dieses Determinativ hat, ist diese Erklärung nicht ganz so deutlich.

Ganz anders ist die Lage bei $d \check{s} r$. Oben wurde bereits festgestellt, daß es ohne weiteres die anderen „Rot"-Wörter ersetzen kann. Sein Anwendungsbereich ist praktisch unbegrenzt. Es kommt etwa mit folgenden Sachbezügen vor: Karneol (Wb. V 488, 5); Natron (488, 6); Myrrhe (488, 8); Holz $(488,9)$; Fett $(488,10)$; Öl $(488,11)$; Getreide (besonders zur Bierherstellung) $(488,12)$; Rinder $(488,14-15)$; Kälber $(488,16)$; Haarfarbe $(489,4$, vgl. unten; 489, 18); Blut $(489,8$; dazu 491, 10-492, 1 usw.); hämatitgefärbtes Wassergefäß $(489,10)$; dazu Wb. V 493, 3-11; vgl. Kees, Farbensymbolik, 462); die rote Königskrone $(489,11$; dazu 493, $12-494,3)$; die Wüste $(489,13$; dazu Wb. V 494, 5-13); der Gebel aḥmar bei Kairo $(489,14)$; Feuer (490, 7-13; vgl. 494, 4 ?); die aufgehende Sonne (Kees, Farbensymbolik 448f.); schließlich der Flamingo, der sich als Hieroglyphe für $d \check{r} r$ eignete (Wb. V 487; 9).

Es gibt sicher noch weitere Verwendungsweisen; aber man sieht bereits deutlich, daß es sich bei einer solchen Variationsbreite nur um ein abstraktes Farbwort handeln kann. Den Be- 
reich der Farbwelt, der durch ďs gefaßt wird, kann man nach diesen Belegen etwas genauer bestimmen. Eine Reihe von Farbträgern sind für uns, ,rot", so das Blut, der Karneol, der Flamingo, u. a. $\mathrm{Zu}$ beachten ist aber auch, daß die Ägypter selbst die ,gelbrote" $d \check{s} r$.t-Wüste meist ,gelb" mit „,roter" Innenzeichnung malen: vgl. Lefebvre, JEA 35, 73 mit Anm. 2; Kees, Farbensymbolik, 417, Anm. 11; Dunham, Naga-ed-Dêr Stelae (Nr. 20; Nr. 87). Dieses ,Gelb“ war offenbar auch $d \check{s} r$. ď̌r als Zusatz zum PN, das die Haarfarbe bezeichnet (Ranke, PN II 11 mit Anm. 20), steht im Gegensatz zu km. Da nur diese Opposition besteht, ist ďr im Gegensatz zu ,Schwarz" mit größter Wahrscheinlichkeit „Blond" und nicht unbedingt das ausgefallene ,Rothaarig", das natürlich auch unter $d \check{r} r$ fallen wird. In der Kunst kann ,,blondes" Haar ,gelb" (mit ,,roter Zeichnung) gemalt werden: Per-nēb, 42, Anm. 29.

2. ,Gelb"

Kein Farbwort aufgeführt.

3. , Grün"6

Bei ,grün" — ,grünen“ muß man alle Wörter ausscheiden, die sich nur auf das Wachsen der Pflanzen beziehen. Wie im Deutschen stehen auch im Ägyptischen beide Bereiche in engster Verbindung. Für das AR bleibt nur der Stamm w3d als Farbwort übrig; der einzige in der älteren Zeit für ,grünen" noch belegte Stamm, $3 h$ ( $s$ 3h $3 h$ Wb. IV 24, 15) ist sicher kein Farbwort.

w3 $\underline{d}$ (Wb. I 264, 12-14), grün, grün sein" mit mehreren nominalen Ableitungen (Wb. I 263 bis 270) steht bei folgenden Sachbezügen: Papyrus (Wb. I 263, 7-264, 4); Pflanzen $(264,12 ; 270,11)$; Feld $(264,13)$; Malachit und anderen grünen Steinen (Wb. I 267, 3-8; Kees, Farbensymbolik, 430; Harris, Lexicographical Studies in Ancient Egyptian Minerals, 104); grüne Augenschminke (Wb. I 267, 9-15); ein Zeugstoff (268, 10-12); Uräusschlange (268, 17-269, 3); das Meer (269, 12-16); wohl auch der Himmel (s. Ke es, Farbensymbolik, 430: ,Als Lichtfarbe kommt ... das Grün dem Lapislazuliblau des Himmels nahe. So kennen die religiösen Texte am Himmel neben dem naturhaften „Binsengefilde“ ein ,Türkisgefilde“ oder ,Türkisteiche“ als Jenseitsland, das man mitunter auch einfach das ,grüne Gefilde" nennen mochte.").

W3 $\underline{d}$ ist nach den vielfältigen Sachbezügen ein abstraktes Farbwort. Es ist leicht zu erkennen,

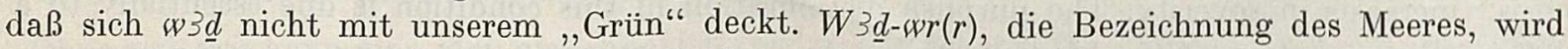
zwar üblicherweise mit ,, das große Grün" oder ,,der große Grüne" übersetzt, ist aber nach dem deutschen Sprachgebrauch richtiger als ,,das große Blau“, ,der große Blaue“ zu übersetzen: wenn wir ein Farbwort auf das Meer anwenden, ist es im Normalfall ,blau" und nicht, grün“. Falls auch der Himmel als w $3 \underline{d}$ belegt ist, wird der Bezug zu unserem ,Blau“ noch deutlicher.

4. , $\mathrm{Blau}$ “6

jrtjw (Wb. I 116, 10-12) steht mit keinem bestimmten Farbton in Verbindung; s. Kees, Farbensymbolik, 465f.; 474. Das andere Wort, das für „Blau“ noch in Frage kommt, ist hasbd. (Wb. III 334, 15-335, 1). Ein abstraktes Farbwort kann hier nicht vorliegen, da der Sachbezug zum Lapislazuli (Wb. III 334, 1-13) auf der Hand liegt. Der Lapislazuli hätte natürlich auch zur Angabe einer Farbe dienen können, aber tatsächlich bedient man sich dieses Mittels nur bei Göttern und ihren Körperteilen, wo es vielleicht sogar richtiger ist, neutral ,lapislazuli-artig“" zu übersetzen.

Ein Wort für ,Blau“" ist im Wb. nicht aufzutreiben. Es läßt sich darüber hinaus eine positive Angabe machen: lapislazuli-blaue (oder -artige) Augen sind ,grün"-gemachte ( $s(3 \underline{d}$ ) Augen: Kees, Farbensymbolik, 465. Der Bereich unseres, Blau“ wird also mit $w 3 d$ wenigstens z. T. gefaßt. Kees bemerkt in seiner Farbensymbolik, 464 und 467, ausdrücklich, daß die ,blaue“ Farbe in der Symbolik der religiösen Texte keine Rolle spielt. Die Erklärung liegt auf der Hand: für den Ägypter gab es kein spezielles ,Blau“, weil es in seiner Sprache kein Wort dafür gab.

5. ,Sehwarz"

$\underline{d}^{c} b$, schwärzen“ (Wb. V 536, 4-6) kann leicht als sachgebunden abgetan werden. Die Ableitung von $\underline{d}^{c} b . t$, ,Holzkohle“ (Wb. V 536, 8-17) ist evident; es liegt auch kein Grund dafür vor, daß $\underline{d}^{c} b$,schwärzen" ohne diesen Sachbezug je gebraucht worden wäre. 
Für ,Schwarz" kommt also nur $\mathrm{km}$ mit seinen Ableitungen in Frage: $\mathrm{km}(\mathrm{m})$ (Wb. V 123,1 bis 124, 8); km ,das Schwarze" (Wb. V 124, 9-12); skm, schwarz werden lassen“ (Wb. V 318, 2-3). Allerhand Dinge können $k m$ sein: Granit (Wb. V 123; dazu 128, 2); Feuerstein $(123,6)$; Bäume $(123,7)$; Hölzer $(123,8)$; Spelt $(123,10)$; Rinder $(123,12$; dazu 125, 5-9); Schafe $(123,13)$; Kälber $(123,14)$; die Eidechse $(123,16)$; das Schwarze im Auge $(123,21$; dazu 124, 13); der sonnengebrannte Erntearbeiter (124, 2); das Haar (123, 18; 124, 7; Ranke, PN II 11 mit Anm. 20); das ägyptische Fruchtland (126, 7-127, 20).

Offensichtlich liegt ein abstraktes Farbwort vor. Deutlich ist auch, daß $\mathrm{km}$ nicht einfach „schwarz" ist: Granit z. B. ist eher ,grau"; der Erntearbeiter „,braun“ oder ,,rotbraun"; in äthiopischer Zeit ist auch die Nacht einmal als km, d. h. ,dunkel", bezeichnet (Wb. V 124 10). Das "Grau"werden der Haare, skm (Wb. IV 317, 12-318, 1) gehört kaum hierher, da ja die Haare sicherlich heller werden. Wie das Wb. vermutet, wird ein Zusammenhang mit skm ,vollenden" usw. (Wb. V 317, 2-11) bestehen und nicht mit skm „,schwärzen“.

6. "Weiß"

$h \underline{d}$ ist das einzige Wort, das das Wb. als ,Weiß" aufführt, s. besonders Wb. III $206 \mathrm{ff}$. $h \underline{d}$ steht in vielfältigen Sachbezügen; so kann man es verwenden bei: Milch (Wb. III 206, 15); Honig $(206,17)$; Kalkstein (I 97, 12); Sandstein (I 97, 13); Holz (III 207, 1); der Säbelantilope ( $m$ 3-ḥd: III 207, 5); Zähnen (III 207, 3; 14); Leinen (III 207, 6); Kleidern (207, 7; 13; 17); Brot (III 207, 9); Silber (III 209, 9-210, 6); der Krone von Oberägypten (III 211, 3-7); und anderswo.

hd ist ein abstraktes Farbwort. Es steht aber auch bei Naturerscheinungen, die zeigen, daß es mit ,weiß" allein nicht richtig gefaßt ist. Besser kann man es mit ,hell" wiedergeben, da es mit dem Tagesanbruch und dem Licht in Zusammenhang steht: Wb. III 207, 20-208, 17. Der Sandstein ist zweifellos auch nicht, ,weiß", sondern ,hellbraun" 0 . ä. Das Silber läßt sich ebenfalls unter „weiß" nicht unterbringen. Die Hieroglyphe für $h \underline{d}, \hat{\phi}$, wird zwar gewöhnlich mit ,weißem“ Kopf gemalt, gelegentlich aber auch mit, gelbem": Per-nēb, 47.

7. Die abstrakten Farbwörter

Die abstrakten Farbwörter heben sich dadurch von anderen Farbbezeichnungen ab, daß sie primär verbal gefaßt sind. Nominale Formen sind davon erst abgeleitet. Von den oben aufgeführten sachgebundenen Wörtern gibt es keine solchen intransitiven Verben ( ${ }^{c} b$ ist transitiv). Ganz im Gegensatz zu den deutschen Farbwörtern, die zunächst als Adjektive vorliegen (Weisgerber, Die Muttersprache im Aufbau unserer Kultur, 1957², 130-132), liegen im Ägyptischen die Verben zugrunde.

Diese Grundverben sind:

a) $d \check{s} r$ Inf. I *dáš $\sim r$, rot sein“ > ropщ CD $432 \mathrm{a}$.

Inf. II *dušăr ,rot sein" > тpoyㅡ CD 432 (nur S).

PPA *dáš $r$, ,rot (seiend)" $>$ Tup

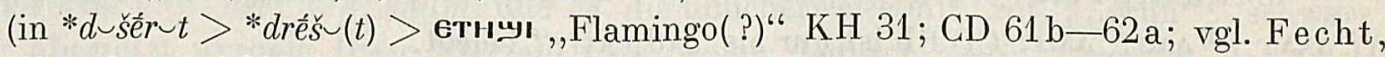
Wortakzent $§ 206$ I a 1, liegt, wenn die Zurückführung auf $d \check{r}$ überhaupt richtig ist, kaum das feminine Partizip vor, da dies bei $w 3 \underline{d}$ vermutlich erhalten ist, und dort anders aussieht.

Zur Form des PPA: AäG § 627; Fecht, Wortakzent § 98, Anm. 158; § 226.

Wie die Form n-тнре CD 432 b einzuordnen ist, bleibt unklar.

b) $w 3 \underline{d}$ Inf. *wáa $3 \underline{d}$, grün sein $">\operatorname{orcot~CD~} 493$.

PPA im Maskulinum nicht erhalten, liegt aber im Femininum wohl in oro(o)re ,greens, herbs" CD 493 b vor:

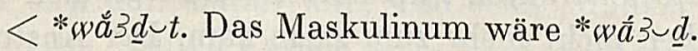

Zur Form des PPA s. bei $d s ̌ r$.

c) $k m(m)$ Inf. $* k \sim m a ̆ m$, ,schwarz sein, werden" $>$ kuou CD $109 \mathrm{~b}$.

PPA *kĕ́m > kau ,schwarz (seiend)"CD 109b. Das Femininum ist erhalten in кнue „Ägypten" CD 110 a $<* k e ́ m \sim t$. 
Zur Form des PPA: ÄäG $\$ 628 \mathrm{bb}$ ): die IIae-gem. wiederholen den zweiten Radikal nicht; vgl. weiter das PPA der 2-rad. Verben: statt min und rih könnte man auch mén und rĕh̆ ansetzen. S. auch Fecht, Wortakzent § 72, Anm. 128.

Die beiden anderen Nominalformen, die im Koptischen erhalten sind, SA kusé,

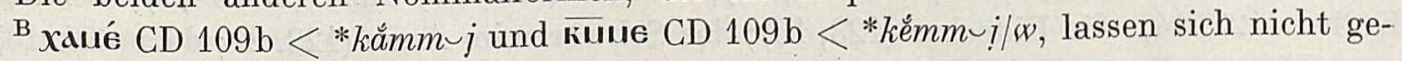
nauer einordnen; vgl. Fecht, Wortakzent, 14.

d) $h \underline{d}$ : Im Koptischen ist $\underline{h} \underline{d}$ nicht mehr das normale Wort für ,,weiß“. Erhalten ist es nur noch als ,Adjektiv" und als ,Silber".

PPA *hẹ̌d $>$ ast , weiß" (seiend)" CD 713 b. Zum e-Vokal und zum Ansatz des PPA s. bei $k m(m)$.

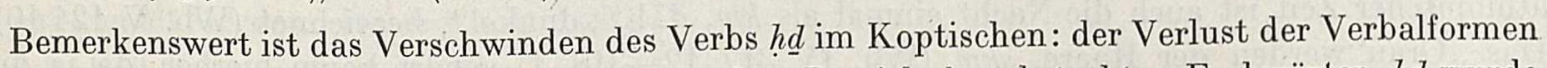
geht Hand in Hand mit der Verdrängung aus dem Bereich der abstrakten Farbwörter. $h \underline{d}$ wurde offenbar mehr und mehr auf das ,Silber" eingeschränkt. Zum Ersatzwort, das in die entstehende Lücke einsprang, s. den folgenden Abschnitt.

\section{Exkurs: die Farbwörter im Koptischen}

Bei der vorausgehenden Liste, die die abstrakten Farbwörter der älteren Zeit herausarbeiten sollte, kam es in erster Linie darauf an, quantitativ zu bestimmen, wie groß der Bestand an diesen Wörtern ist. Zur genaueren Bestimmung der Einzelheiten konnte aber leicht auf später belegtes Material gegriffen werden, wenn auch älteres bevorzugt wurde: daß die Vierteilungen der Farberscheinungen erst sehr spät verändert wurde, zeigen die abstrakten Farbwörter des Koptischen. Till hat in den Studia Biblica et Orientalia $3(1959)=$ Analecta Biblica 12, S. 331-342 einen Aufsatz veröffentlicht mit dem Titel: Die Farbenbezeichnungen im Koptischen. Leider hat er das eigentliche Problem der Farbwörter, nämlich das der gegenseitigen Abgrenzung, nicht berührt; im Prinzip wird nicht mehr geboten, als was sich über den englischen Index aus dem CD herausholen läßt, d. h. eine Projektion der koptischen Farbwörter auf unsere Übersetzungsgewohnheiten. In unserem Zusammenhang sind nur die Wortstämme wesentlich, nicht alle Einzelformen (zu diesen s. Till).

Genau wie im Ägyptischen hat man 1. Tcop» usw. CD 432; 2. orcot usw. CD 493; 3. kHou usw. CD 109b. 4. Einer eingehenderen Besprechung bedarf hd. Wie wir in Abschn. K 7 bereits gesehen haben, hat es im Koptischen seinen verbalen Bereich eingebüßt. Dazu kommt, daß a৯T meist das Silber bezeichnet, selten nur „Weiß“ (vgl. Till, 335). Als abstraktes Farbwort scheidet $\underline{h d}$ also aus. Was ist aber dann im Koptischen an die Stelle des alten $h \underline{d}$ getreten? Für unser ,Weiß" findet man, abgesehen von dem griechischen $\lambda \varepsilon u x o ́ s$ (s. Till 335), noch zwei weitere Wortstämme:

a) Inf. $\mathrm{SA}_{2} \mathrm{~B}$ Ordy, ${ }^{\mathrm{A}}$ OrBaz CD $476 \mathrm{~b}$. Das alte PPA ist erhalten als orcoby CD $476 \mathrm{~b}$. Nach dem Formenbestand liegt es nahe, hier den Nachfolger von $h \underline{d}$ zu sehen. Der Wortstamm ist seit der 18. Dynastie belegt: $w b h$ Wh. I 295, 12-296, 2. Die Ausbildung dieses $w b h$ zum abstrakten Farbwort des Koptischen zu verfolgen, führte in unserem Zusammenhang zu weit.

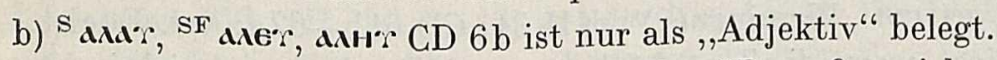

Nach den detaillierten Angaben des CD muß es sich um ein abstraktes Farbwort handeln. Da es aber nicht bis ins Ägyptische zurückverfolgt werden kann, handelt es sich hier zweifellos um das jüngste Farbwort für ,weiß". Auch im Koptischen hat es sich über das $\mathrm{S}$ und $\mathrm{F}$ hinaus nicht verbreitet, noch ist es in literarische Texte eingedrungen (Till, 334). Es besteht damit die Möglichkeit, daß in relativ später Zeit, vielleicht sogar in einem lokal abgeschlossenen Gebiet (vgl. zu einer entsprechenden Dialektlokalisierung zuletzt: Morenz, Handb. der Orientalistik, Abt. I, Bd. I, Abschn. I, 102f.; Vergote, CdE 36 [1961] 243-245), eine Tendenz bestand, die abstrakten Farbwörter vom verbalen Bereich zu lösen, die aber bei den anderen Farben nicht mehr zum Zuge kam. Der Anstoß zu einer solchen Loslösung könnte bei ,weiß" besonders naheliegen: es bestünde die Möglichkeit, auf diese Art die verbal gefaßten Lichterscheinungen von den neu ,,adjektivisch“ gefaßten Farberscheinungen zu trennen. Dies nachzuweisen liegt außerhalb dieser Untersuchung. 
Die einzige wirkliche Neuerung, die eine Differenzierung der Farbskala mit sich bringt, scheint ein Farbwort im Bereich „Rot-Gelb“ zu sein, Inf. upow CD 183b, dazu ein ,Adjektiv“ SBF uнpщy, SF uepu, SA upu, CD 183b. Dieses Verbum, wohl aus der alten Farbsubstanz mnš.t gebildet, (vgl. Iversen, Some Ancient Egyptian Paints and Pigments, $31 \mathrm{f}$.), ist zuerst in griechischer Zeit belegt: mrš Wb. II 113, 1; demotisch in römischer Zeit: Erichsen, Glossar, 170. Wie die anderen abstrakten Farbwörter (außer dem noch späteren dגa) hat es einen voll ausgebauten Verbalbereich. Das „Adjektiv“" läßt sich mit 1-тнрщ vergleichen. Es gibt aber noch eine andere Erklärungsmöglichkeit: da in griechischer Zeit die Partizipien kaum mehr bildbar waren, sondern wohl nur noch in erstarrten Resten, wie z. B. in den Farb-,,Adjektiven“, vorlagen, könnte die e-Vokalisation entweder in Analogie zu anderen ,Adjektiven“ (etwa *rнрщ) oder aber nach der Farbsubstanz $m n s ̌ . t$ gebildet worden sein, die dann als *mĕrš $(t)<* m e ̌ n s ̌ v t$ anzusetzen wäre. Wie sich rop:y/тpoy usw. und upoy usw. im Koptischen gegenseitig abgrenzen, muß weiterer Untersuchung überlassen bleiben.

Zur Angabe genauerer Farbnuancen hilft man sich mit Sachbezügen; so steht arau ume CD

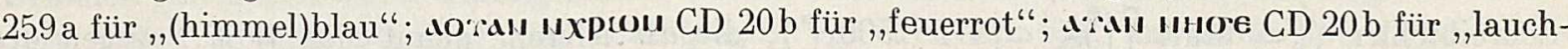
grün"; wraw unorept CD 20 b für ,,rosenfarbig, rosa"; usw. (vgl. auch Till, loc. cit. $341 \mathrm{f}$.)
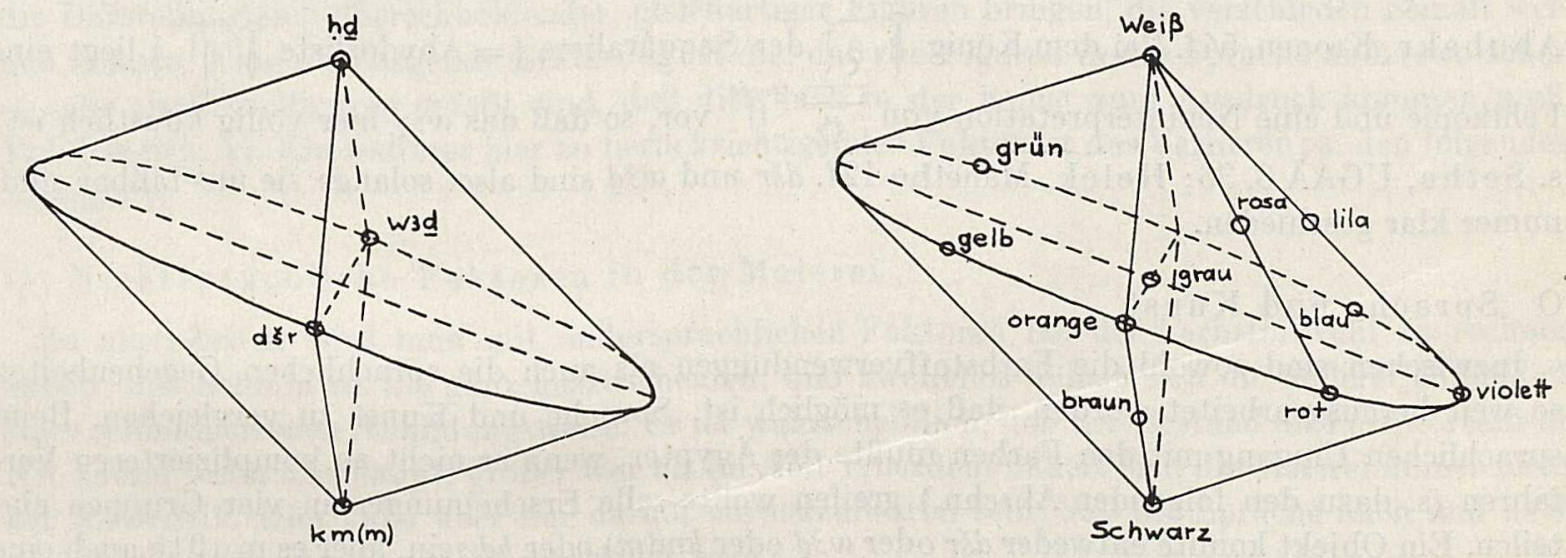

Fig. 4

Es sieht so aus, als ob vom AR bis in die Spätzeit das gleiche Farben-,,Feld" (s. dazu den folgenden Abschn.) gegolten hätte.

\section{Das ägyptische Farbenfeld}

Die Zusammenstellung in Abschnitt H K hat gezeigt, daß es im (Alt-)Ägyptischen vier abstrakte Farbwörter gibt: $d \check{s} r ; w 3 \underline{d} ; k m(m)$ und $h \underline{d}$, die üblicherweise mit ,,rot"; , ,grün“; ,,schwarz" und ,weiß" übersetzt werden. Diese Vierzahl kann weiter dadurch gestützt werden, daß in der Farbensymbolik der religiösen Texte vier Farben eine Rolle spielen, die meist in den Texten auch mit den vier abstrakten Farbwörtern gefaßt sind.

Da diese vier Farbwörter ,,abstrakt" sein müssen, ist zu fordern, daß mit ihnen die gesamte dem Auge zugängliche Farbwelt eingefangen werden kann, d. h., daß diese vier Farbwörter ein geschlossenes ,Wortfeld“ bilden (vgl. L. Weisgerber, Vom Weltbild der deutschen Sprache, 1. Halbband $\left.{ }^{2}, 127-133\right)$. Die Gliederung im Modell des schiefen Doppelkegels bietet sich an: $k m(m)$ und $h \underline{d}$ teilen die Helligkeitsskala in zwei Bereiche auf; $d \breve{r} r$ und $w 3 \underline{d}$ teilen den ,Farbtonkreis" unter sich auf. $k m(m)$ wäre also etwa ,dunkel", hִ $\underline{d}$, ,hell“; $d \check{s} r$ bezeichnete die ,warmen Farben", w3 3 die ,kalten Farben".

Wie sich die ägyptischen Farbwörter zu den deutschen verhalten, d. h. wie sie übersetzt werden müssen, ergibt sich leicht, wenn man den Doppelkegel des ägyptischen Farbenfeldes dem Doppelkegel des deutschen Farbenfeldes gegenüberstellt (schematisch in Fig. $4 \mathrm{a}$ und b; zum Deutschen vgl. L. Weisg erber, Vom Weltbild der deutschen Sprache, 1. Halbband $\left.{ }^{2}, 132\right)$. 
dšr dürfte etwa sein: ,,rot, orange, gelb, (hell)braun, rosa (violett, lila).“

w3 3 ist etwa: ,grün, blau, violett, lila“.

$k m(m)$ entspräche etwa: ,schwarz, grau, (dunkel)braun, violett.“

$h \underline{d}$ wäre ungefähr: ,,weiß, grau (gelb, grün).“

Für die Entstehung der ägyptischen, viergeteilten Farbwelt läßt sich mit Wahrscheinlichkeit die Quelle angeben: die ägyptische Landschaft mit den vier lebenswichtigen Faktoren: Fruchterde $(\rightarrow k m(m))$; Wüste $(\rightarrow d \check{s} r)$; Pflanzenwelt $(\rightarrow w 3 \underline{d})$ und Tageslicht $(\rightarrow \underline{h d} \underline{d})$.

\section{$\mathrm{N} d \check{s} r$ und $w 3 \underline{d}$}

Gelegentlich schien es, als ob dšr und $w 3 \underline{d}$ als Farbwörter miteinander wechseln könnten. Sethe, Urgeschichte, 160 mit Anm. 2, neigte zu der Ansicht, daß ursprünglich w3 $\underline{d}$ auch den Bereich von $d \check{s} r$ mit umfaßte und erst später eine Aufteilung in w $w \underline{d}$ und $d \check{s} r$ auftrat. Tatsächlich handelt es sich bei dem Belegmaterial z. T. um symbolische Verwendung der Farbwörter; einmal um einen

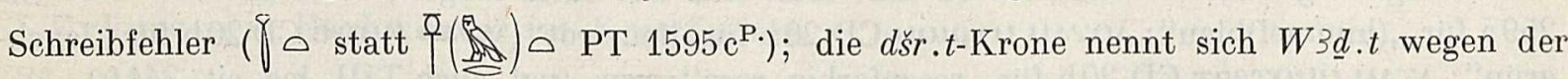
Beziehung zur Uräusschlange. Siehe Kees, Farbensymbolik, 434f.; 439; 431 f.; 458; vgl. auch

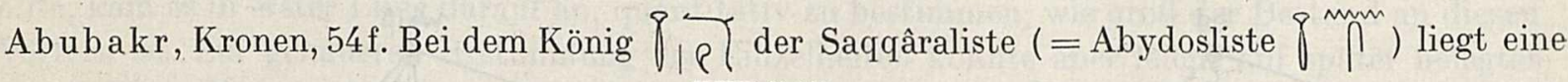
Fehlkopie und eine Neuinterpretation von $\bigcap^{n}$. vor, so daß das $w 3 \underline{d}$ hier völlig künstlich ist;

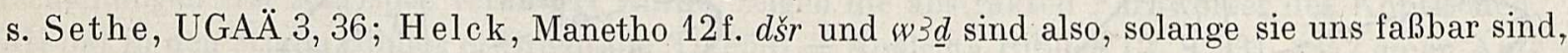
immer klar geschieden.

\section{O Sprache und Kunst}

Inzwischen sind sowohl die Farbstoffverwendungen als auch die sprachlichen Gegebenheiten so weit herausgearbeitet worden, daß es möglich ist, Sprache und Kunst zu vergleichen. Beim sprachlichen Umgang mit den Farben mußte der Ägypter, wenn er nicht zu komplizierteren Verfahren (s. dazu den folgenden Abschn.) greifen wollte, alle Erscheinungen in vier Gruppen einteilen. Ein Objekt konnte entweder $d \breve{s} r$ oder $w 3 \underline{d}$ oder $k m(m)$ oder $h \underline{d} \underline{d}$ sein, aber es $m u ß t$ te auch eine dieser Farben tragen. War nun dem ägyptischen Künstler die Aufgabe gestellt, diese viergeteilte Welt mit seinen Farbstoffen darzustellen, kam er in Konflikt: denn seine Farbstoffe deckten sich nicht mit seiner sprachlichen Farbwelt und konnten sich auch nicht damit decken, da die Zahl der zur Verfügung stehenden Farbstoffe nicht direkt sprachabhängig ist, sondern in erster Linie von den zur Verfügung stehenden Rohstoffen und den chemischen und technischen Kenntnissen abhängt. Tatsächlich waren sechs (oder mehr) Farbstoffe zu verwenden. Wenn also z. B. dšr zu malen war, so standen wenigstens „Rot" und "Gelb" zur Wahl, sollte $w 3 \underline{d}$ gemalt werden, so wenigstens „Grün“ und „Blau“. Dazu kommt, daß jedes Farbwort von vornherein eine große Zahl von Farbnuancen umfaßt, der Farbstoff dagegen relativ starr ist. Daß also ein bestimmtes Naturvorbild in der Malerei einmal mit dem einen Farbstoff erscheint, das andere Mal mit dem anderen, war zwangsläufig. Mag man dem Ägypter des AR auch unterstellen, er habe früh versucht, aus diesem Dilemma dadurch herauszukommen, daß er seine Farbstoffe mehr nach dem Scheineindruck, also in koloristischem Verfahren auswählte, so konnte einem solchen Vorgehen bei dem Grundcharakter der Kunst doch kein dauernder Erfolg beschieden sein, da die Sprache sich immer von neuem mit ihrem Recht geltend machte. Die Tatsache des Farbwechsels ist ja dann auch nach dem oben gegebenen Befund in die Augen springend.

Es bietet sich nun auch eine Erklärung für das in Abschn. H gefundene unterschiedliche Verhalten der Nachbarfarbpaare an: die Unregelmäßigkeit hängt unmittelbar mit dem Aufbau des ägyptischen Farbenfeldes zusammen: die Grenzen zwischen den Farbwörtern liegen an den Stellen, wo die Vertauschbarkeit der Farben niedrig ist (zwischen ,Gelb" und ,Grün“; ,,Rot" und „Blau“); wo die Vertauschbarkeit hoch ist, fehlen im Ägyptischen die Grenzscheiden bei den Farbwörtern. Das heißt also: konnte der Ägypter mit seinen Farbwörtern zwei Farbtöne auseinander- 
halten, so war die Konkurrenz der entsprechenden Farbstoffe zurückgedrängt, die Farbstoffwahl weniger dem Zufall überlassen.

\section{P Der Einfluß anderer Sprachmittel in der Malerei}

War soeben nachgewiesen worden, daß die speziellen Farbwörter hinter der Tätigkeit des Malers stehen, so ist es doch möglich, wenn auch nicht das Normale, daß andere Sprachmittel bei der Farbstoffverwendung eine Rolle spielen. Sicher hatte jede Farbsubstanz ihren Namen. Es könnte sich also in gewissen Fällen für bestimmte Dinge, die in der Sprache auch gewortet waren, eine Konvention herausgebildet haben, diese Gegenstände immer mit dem gleichen Farbstoff zu malen. Ein guter Beleg dafür ist die Praxis, die Hautfarbe ägyptischer Männer durchweg „rot", die ägyptischer Frauen durchweg, ,gelb“ wiederzugeben. In den Farbwörtern läßt sich dafür keine Begründung finden. Aber in der gesamten ägyptischen Sprachwelt ist der Unterschied zwischen Mann und Frau derart verankert, daß eine solche verschiedene Behandlung beim Bemalen mit Leichtigkeit durchgehalten werden konnte. Selbst die Stelen aus Nag ed-Dêr (s. dazu unten Abschn. R) sind hier konsequent. Vgl. Per-nēb, 65f. mit Anm. 166 (Der Erklärungsversuch auf S. 65, Anm. 171 ist sicher auszuscheiden). Als zweites Beispiel kann man vielleicht die Darstellung sich überschneidender, gleichartiger Figuren bringen, die verschieden bemalt werden können. Eine naheliegende Erklärung ist die, daß die Figuren in der Sprache anderswo schon so sehr als Einzelfiguren gefaßt sind, daß dies auch in der Kunst zum Ausdruck kommen muß. Vgl. Per-nēb, 44. Ein weiterer hier zu berücksichtigender Faktor ist das Variieren; s. den folgenden Abschnitt.

\section{Q Nichtsprachliche Faktoren in der Malerei}

$\mathrm{Zu}$ allen Zeiten wird man mit außersprachlichen Faktoren bei der Farbstoffwahl zu rechnen haben: das Sehbild ist nie ganz auszuscheiden, und zweifellos nähert sich die Malerei allmählich einer sehbildgetreuen Abbildungsweise. Es ist wahrscheinlich, daß der Bestand an Farbwörtern im NR kaum, wenn überhaupt, größer war als im AR. Trotzdem nähert sich die Malerei immer mehr der Koloristik. Dies kann aber nur darauf zurückzuführen sein, daß die Sprache nach und nach von ihrer beherrschenden Rolle verdrängt wird.

Schließlich ist mit Impulsen ganz anderer Art zu rechnen, die zweifellos über die Sprache hinaus auf ursprünglichere Schichten hinweisen. Wenn mehrere gleichartige Formen sich wiederholen müssen, ist es zu viel, sie auch noch gleich anzumalen. Um diesem Übel der Gleichförmigkeit abzuhelfen, wird die Farbe variiert. Vergleiche die drei $\circlearrowright$ in Per-nēb, pl. I a (,,blau“; ,,rot" ; ,,blau“); g (,,blau“; ,grün“; ,,blau“). Es handelt sich hier um etwas wie das ästhetische oder rhythmische Empfinden des Ägypters, Kräfte also, die zwar auch in der Sprache wirken können, die aber außerhalb der Sprache ihren Beweggrund haben.

Die Abgrenzung sprachlicher und nichtsprachlicher Faktoren in der Kunst wird im einzelnen noch genauester Untersuchungen bedürfen.

\section{R Anhang: Nag'c ed-Dêr}

Eine kurze Bemerkung soll den Stelen aus Nag`c ed-Dêr gelten, die D unham veröffentlicht hat. Soweit die Hieroglyphen auf den Stelen überhaupt polychrom ausgemalt sind, liegt, ganz abgesehen davon, daß „Grün“ und „Blau“ nur Varianten sind, also nicht in Opposition stehen, eine derartige Unüberlegtheit bei der Farbstoffwahl (nach der Statistik) vor, daß man nichts daraus ablesen kann: so ziemlich alles kann mit allem vertauscht werden. Es ließe sich also allein auf Grund der Farbbehandlung die Qualität der Stücke (dann auch der Einzelstücke) bestimmen. Hier hat offenbar selbst die Sprache bei der Herstellung der „Kunstwerke" nicht mehr funktioniert: ein Teil dieser „Künstler" hat sich also bei der Arbeit gar nichts mehr gedacht, sondern einfach drauflosgepinselt. 